Sharif University of Technology
Scientia Iranica
SCIENTIA

\title{
Developing a finite element beam theory for nanocomposite shape-memory polymers with application to sustained release of drugs
}

\author{
M. Baghani $i^{a, *}$, R. Dolatabadi ${ }^{b}$ and M. Baniassadi ${ }^{a}$ \\ a. School of Mechanical Engineering, College of Engineering, University of Tehran, Tehran, Iran. \\ b. Department of Drug and Food Control, Faculty of Pharmacy, Tehran University of Medical Sciences, Tehran, Iran. \\ Received 29 June 2015; received in revised form 19 December 2015; accepted 27 February 2016
}

\author{
KEYWORDS \\ Shape-memory \\ polymers; \\ Finite element; \\ Smart beam; \\ Euler-Bernoulli beam \\ theory; \\ nano/micro \\ reinforcement.
}

\begin{abstract}
In this paper, a thermodynamically consistent constitutive model, recently proposed for nanocomposite Shape-Memory Polymers (SMPs), is used as a basis for development of SMP beam element in a finite element framework. The beam theory utilized here is the Euler-Bernoulli beam theory with its basic assumptions. Effects of different materials as well as the geometric structural parameters, e.g. reinforcement (nano/micro-particles) volume fraction, viscosity coefficients, and external loads, on the thermomechanical response of the structure are studied in this work. The beam element numerical results are compared to those of $3 \mathrm{D}$ finite element modeling to verify validity of the beam element formulation and the assumptions made therein. This beam element provides us with a fast and reliable tool for simulation of structures, consisting of reinforced SMP beams. As an application, the developed nanocomposite SMP beam element could be used for numerical modeling of thermomechanical response of the drugs (e.g., theophylline) coated by films of SMP nanocomposites. It is shown that the numerical results are in correspondence with those of the experiments reported for sustained release of SMPnanocomposite based drugs.

(C) 2017 Sharif University of Technology. All rights reserved.
\end{abstract}

\section{Introduction}

Shape-memory materials offer a promising perspective for materials science and engineering, providing access to extensive unconventional functions in various classes of materials: metals, ceramics, and polymers [1]. These materials are capable of returning from a fixed shape to their original shape. This class of materials have been researched, developed, and used in a wide range of applications, such as advanced technologies in the aerospace and medical-related industries [25]. The Shape-Memory Effect (SME) is normally in-

\footnotetext{
*. Corresponding author. Tel.: +98216111 9921; Fax: +98216600 0021

E-mail address: baghani@ut.ac.ir (M. Baghani)
}

duced through external stimuli, e.g. heat, electricity, or magnetism $[6,7]$. The mechanisms controlling the thermally activated SME in Shape-Memory Polymers (SMPs) are different from those that play roles in shape-memory alloys or ceramics. These phenomena result in differences in the thermomechanical responses of the material [8].

However, the much smaller stiffness of unreinforced SMPs limits their practical applications, in which a larger recovery stress is necessary. To get over such limitations, different reinforcement techniques are introduced in the literature [9-13]. The necessity to activate SMPs through non-thermal stimuli has led to employing conductive fillers, e.g. conductive particles, nanocarbon particles, and conductive fibers [14-17]. The SMP composites are considered as a conductive 
polymer with potential practical applications in the fields of micro-sensors and actuators. Thus, different chemical sensors and bio-sensors with fascinating characteristics could be designed and used in medicine for fast bio-diagnostic systems [15,18-20].

It should be noted that multifunctional polymers combining two functions, such as SME and biodegradability [21] or biodegradability and drug release [22], have already been introduced [23]. But, a material that exhibits all these three functions has not been deeply investigated yet. This would let us use SME to perform minimally invasive implantation of quite massive devices, biodegradability to eliminate a second surgery for removal, and controlled drug release during encountering infections [24]. In a very recent study, Kashif et al. [25] investigated the sustained drug release and hydrolytic degradation of biodegradable shape-memory poly( $\varepsilon$-caprolactone)/polyhedral oligomeric silsequioxane nanocomposites. In vitro drug release, investigations of the phosphate buffer solution $(\mathrm{pH}=7.4)$ showed that TspPOSS nanocomposite nanocrystals led to sustained release of the drug for the nanocomposites. PCL/TspPOSS nanocomposite (90 wt/10 wt) containing $10 \mathrm{wt} \%$ drug (Theophylline) demonstrated good thermally activated SME with a shape fixation ratio of $81 \%$ and shape recovery ratio of $85 \%$.

Some researchers also investigated the effect of addition of nano-particles into SMP matrix $[26,27]$. Zhang and $\mathrm{Ni}$ [28] performed some experiments to study the effects of carbon fiber fabric reinforcement on the mechanical behavior of SMP sheets. The resulting SMP based laminates possessed good shape recoverability. They also showed that the bending recovery ratio was high compared to that of the SMP sheet at different recovery durations [28]. Despite a variety of SMP applications involving bending loads, bending characterizations are not much extensive $[29,30]$. Some researchers tried to experimentally characterize the behavior of SMPs [11,30-33]. Up to now, characterization of the SMP behavior has been carried out with uniaxial tests [11,31-34]; however, flexural thermomechanical tests could aid the characterization of an SMP constitutive model.

Proposing an elastic-predictor inelastic-corrector, Ghosh et al. [5] introduced a finite element SMP beam based on a constitutive model already published by the same authors. Baghani et al. [35] presented analytic expressions for deflection of an SMP beam utilizing Euler-Bernoulli beam theory in a thermomechanical SME cycle. They employed the constitutive model already proposed in [36], where timedependent effects were neglected. In another work, Baghani and Taheri [37] extended this method to investigate the mechanical behavior of reinforced SMP beams.
In this paper, we propose finite element solutions for nanocomposite SMP beams in an arbitrary loadingdisplacement recovery cycle. Employing the EulerBernoulli beam theory, a 1D SMP beam element is proposed. This formulation is helpful in simulation of structures that consist of SMP beams. This newly introduced SMP beam element can be utilized for either parametric study (material or geometrical parameters) of the SMP beam behavior or design and optimization involving a large number of simulations in which computational cost is a critical parameter. Making use of this beam element dramatically decreases the computational cost, compared to 3D general element previously proposed by Baghani et al. [38]. We also investigate the SME of SMP nanocomposites to qualitatively study its drug release characteristics.

The paper is organized as follows. Firstly, a 3D constitutive equation for SMPs is briefly reviewed in Section 2. Then, in Section 3, the 3D constitutive relations are reduced to the cases in which EulerBernoulli beams undergo arbitrary bending loadings. In this section, development of the SMP beam finite element is discussed in detail. In Section 4, by employing the newly introduced SMP beam element, some numerical examples are solved. It will be shown that the numerical results are in correspondence with those of experiments reported for sustained release of SMP-nanocomposite based drugs. Results of the present work are compared to those of 3D modeling already published by the same authors as well as some experiments available in the literature. Finally, in Section 5, we present a summary and draw conclusions.

\section{Brief review of the SMP constitutive model development}

Since the shape-memory effect characteristics are discussed in details in some already published papers by the same authors, in this paper, for the sake of brevity, we avoid repeating these details and refer to $[36,38$ 40]. In those papers, the 3D diagrams of stress-straintemperature for both stress-free strain recovery and fixed-strain stress recovery are introduced.

Baghani et al. [36] proposed a constitutive model for SMPs in the small strain regime. They then extended this model to time-dependent loading conditions in [38]; in this work, we employ this model to properly account for thermomechanical response of SMPs. They also extended this model to the domain of large strains employing two different approaches $[40,41]$. As described in $[36,38-40]$, it has been assumed that the rubbery and glassy phases are able to be transformed into each other, through the external stimuli of heat. The evolution laws for internal variables have been derived in an arbitrary thermomechanical loading. They have decomposed the 
strain into four parts as:

$$
\varepsilon=\phi_{p} \varepsilon^{p}+\phi_{h} \varepsilon^{h}+\varepsilon^{i}+\varepsilon^{T}
$$

where superscripts $p$ and $h$ stand for the SMP and hard segments, respectively. Also, $\varepsilon^{i}$ and $\varepsilon^{T}$ are the irreversible and thermal strains, respectively, while $\varepsilon^{p}$ is the strain in SMP segment. $\phi_{p}$ and $\phi_{h}$ denote the volume fractions of the SMP and hard segments, respectively $\left(\phi_{p}+\phi_{h}=1\right)$. Besides, the strain in the SMP segment is decomposed into three parts as:

$$
\varepsilon^{p}=\varphi_{r} \varepsilon^{r}+\varphi_{g} \varepsilon^{g}+\varepsilon^{i s}
$$

where subscripts $r$ and $g$ stand for the rubbery and glassy phases, respectively. Also, $\varphi_{r}$ and $\varphi_{g}$ are volume fractions of the rubbery and glassy phases, respectively $\left(\varphi_{r}+\varphi_{g}=1\right)$. It is noted that $\varphi_{r}$ and $\varphi_{g}$ are temperature-dependent functions, while $\phi_{p}$ and $\phi_{h}$ are constant parameters. $\varepsilon^{i s}$ is the stored strain in the material and evolves in the following form [38]:

$$
\dot{\varepsilon}^{i s}=\dot{\varphi}_{g}\left(k_{1} \varepsilon^{r}+k_{2} \frac{\varepsilon^{i s}}{\varphi_{g}}\right)
$$

where:

$$
\left\{\begin{array}{lll}
k_{1}=1, & k_{2}=0 ; & \dot{T}<0 \\
k_{1}=0, & k_{2}=1 ; & \dot{T}>0 \\
k_{1}=0, & k_{2}=0 ; & \dot{T}=0
\end{array}\right.
$$

where dot denotes differentiation with respect to time. Moreover, to account for the viscoelastic behavior of SMPs, some other decompositions are assumed. In the range of small strains, strains are additively decomposed in the glassy, rubbery, and hard phases as:

$$
\varepsilon^{r}=\varepsilon^{e r}+\varepsilon^{i r}, \quad \varepsilon^{g}=\varepsilon^{e g}+\varepsilon^{i g}, \quad \varepsilon^{h}=\varepsilon^{e h}+\varepsilon^{i h},
$$

in which the superscripts $e \beta$ and $i \beta(\beta=r, g, h)$ denote the elastic and inelastic (viscous)parts of the strain in all phases, respectively. Employing the mixture rule, the free-energy density function, $\Psi$, for an amorphous reinforced SMP is introduced as:

$$
\begin{aligned}
\Psi(\varepsilon & \left., T, \phi_{p}, \phi_{h}, \varphi_{g}, \varepsilon^{r}, \varepsilon^{e r}, \varepsilon^{g}, \varepsilon^{e g}, \varepsilon^{h}, \varepsilon^{e h}\right) \\
= & \phi_{h} \Psi_{h}\left(\varepsilon^{h}, \varepsilon^{e h}\right)+\Psi_{T}(T) \\
& +\phi_{p}\left[\varphi_{r} \Psi_{r}\left(\varepsilon^{r}, \varepsilon^{e r}\right)+\varphi_{g} \Psi_{g}\left(\varepsilon^{g}, \varepsilon^{e g}\right)\right] \\
& +\Psi_{\lambda}\left(\varepsilon, T, \phi_{p}, \phi_{h}, \varphi_{r}, \varphi_{g}, \varepsilon^{r}, \varepsilon^{g}, \varepsilon^{i s}, \varepsilon^{h}, \varepsilon^{i}\right)
\end{aligned}
$$

in which $\Psi_{r}, \Psi_{g}$, and $\Psi_{h}$ are Helmholtz free-energy density functions of the rubbery, glassy, and hard phases, respectively. Temperature is denoted by $T$ and the thermal energy is represented by $\Psi_{T}$. Besides, to satisfy Eqs. (1), (2), and (4) in the formulation, using the method of Lagrange multipliers, the term $\Psi_{\lambda}$ is added into the free energy as:

$$
\begin{aligned}
\Psi_{\lambda} & \left(\varepsilon, T, \phi_{p}, \phi_{h}, \varphi_{r}, \varphi_{g}, \varepsilon^{r}, \varepsilon^{g}, \varepsilon^{i s}, \varepsilon^{h}, \varepsilon^{i}\right) \\
& =\lambda:\left[\varepsilon-\phi_{p}\left(\varphi_{r} \varepsilon^{r}+\varphi_{g} \varepsilon^{g}+\varepsilon^{i s}\right)-\phi_{h} \varepsilon^{h}-\varepsilon^{i}-\varepsilon^{T}\right],
\end{aligned}
$$

in which $\boldsymbol{\lambda}$ is the Lagrange multiplier. They also defined:

$$
\begin{aligned}
& \Psi_{\beta}\left(\varepsilon^{\beta}, \varepsilon^{e \beta}\right)=\Psi_{\beta}^{\mathrm{eq}}\left(\varepsilon^{\beta}\right)+\Psi_{e \beta}^{\mathrm{neq}}\left(\varepsilon^{e \beta}\right), \\
& \beta=r, g, h,
\end{aligned}
$$

in which the superscripts ' $\mathrm{eq}^{\prime}$ and ' $\mathrm{neq}^{\prime}$ are the equilibrium and non-equilibrium parts of $\Psi_{\beta}\left(\varepsilon^{\beta}, \varepsilon^{e \beta}\right)$. In this model, it is emphasized that the internal variables are $\varphi_{g}, \varepsilon^{i r}, \varepsilon^{i g}, \varepsilon^{i h}, \varepsilon^{i}$, and $\varepsilon^{i s}$. Thus, evolution laws for the internal variables in the context of continuum thermodynamics should be introduced.

Satisfying the second law of thermodynamics in the form of Clausius-Duhem inequality gives:

$$
\begin{aligned}
\boldsymbol{\sigma} & =\frac{\partial \Psi_{r}^{\mathrm{eq}}}{\partial \varepsilon^{r}}+\frac{\partial \Psi_{r}^{\mathrm{neq}}}{\partial \varepsilon^{e r}}=\frac{\partial \Psi_{g}^{\mathrm{eq}}}{\partial \varepsilon^{g}}+\frac{\partial \Psi_{g}^{\mathrm{neq}}}{\partial \varepsilon^{e g}} \\
& =\frac{\partial \Psi_{h}^{\mathrm{eq}}}{\partial \varepsilon^{h}}+\frac{\partial \Psi_{h}^{\mathrm{neq}}}{\partial \varepsilon^{e h}}
\end{aligned}
$$

Furthermore, in accordance with the viscoelastic behavior of polymers, the following evolution laws are sufficient conditions for satisfaction of Clausius-Duhem inequality [38]:

$$
\begin{aligned}
\dot{\varepsilon}^{i r} & =\frac{1}{\eta_{r}} \frac{\partial \Psi_{r}^{\text {neq }}}{\partial \varepsilon^{e r}}, & \dot{\varepsilon}^{i g} & =\frac{1}{\eta_{g}} \frac{\partial \Psi_{g}^{\text {neq }}}{\partial \varepsilon^{e g}}, \\
\dot{\varepsilon}^{i h} & =\frac{1}{\eta_{h}} \frac{\partial \Psi_{h}^{\text {neq }}}{\partial \varepsilon^{e h}}, & \dot{\varepsilon}^{i} & =\frac{1}{\eta_{i}} \sigma,
\end{aligned}
$$

in which $\eta_{r}, \eta_{g}, \eta_{h}$, and $\eta_{i}$ are viscosity coefficients of the rubbery, glassy, hard, and irreversible parts, respectively.

\section{Development of an SMP Euler-Bernoulli beam element}

In this section, it is assumed that equilibrium and nonequilibrium elastic strain energies are in the forms of $\Psi_{\beta}^{\mathrm{eq}}\left(\varepsilon^{\beta}\right)=\frac{1}{2} \varepsilon^{\beta}: \mathbb{K}_{\beta}^{\mathrm{eq}}: \varepsilon^{\beta}$ and $\Psi_{\beta}^{\mathrm{neq}}\left(\varepsilon^{e \beta}\right)=\frac{1}{2} \varepsilon^{e \beta}$ : $\mathbb{K}_{\beta}^{\text {neq }}: \varepsilon^{e \beta}, \beta=r, g, h$, in which $\mathbb{K}_{\beta}^{\mathrm{eq}}$ 's and $\mathbb{K}_{\beta}^{\text {neq }}$ 's are fourth-order elasticity tensors corresponding to each phase.

Following the general procedure presented by Reddy [42], we employ the basic assumptions of EulerBernoulli beam theory, in which the bending of beams 
with small strains and rotations is found from the following displacement field:

$$
u_{1}=-z \frac{d w}{d x}, \quad u_{2}=0, \quad u_{3}=w(x),
$$

where $u_{1}, u_{2}$, and $u_{3}$ are the displacements of a material point at the location $(x, y, z) . u$ and $w$ are the axial and transverse displacements of the geometric centroid of the cross section on the $x$-axis. An additional assumption has also been made: Transverse loads only cause transverse displacement and no shear strain is generated. Thus, the following linear strain is obtained:

$$
\varepsilon_{x x}=-z \frac{d^{2} w}{d x^{2}} .
$$

Since we aim to develop Euler-Bernoulli beam element for SMPs, the constitutive model presented in Section 2 should be reduced to the case where only axial strain (along beam axis) exists. Moreover, we should present the time-discrete form of the SMP constitutive model. We subdivide the time interval of interest $[0, t]$ in subincrements and solve the nonlinear problem over the generic interval $\left[t_{n}, t_{n+1}\right]$ with $t_{n+1}>t_{n}$. For the sake of simplicity, we indicate with the subscript $n$ a quantity evaluated at time $t_{n}$ and with no subscript a quantity evaluated at time $t_{n+1}$. We also show the time increment by $\Delta t$. Knowing the solution and the strain $\varepsilon_{n}$ at time $t_{n}$, as well as the strain $\varepsilon$ at time $t_{n+1}$, the stress and the internal variables should be updated from the deformation history. Therefore, the timediscrete implicit form of the SMP constitutive model, specifically in Eq. (3), yields:

$$
\begin{aligned}
& \varepsilon^{i s}=\varepsilon_{n}^{i s}+k_{1} \Delta \varphi \varepsilon^{r}+k_{2} \varphi^{-1} \Delta \varphi \varepsilon^{i s} \\
& \Rightarrow \varepsilon^{i s}\left(1-k_{2} \varphi^{-1} \Delta \varphi\right)=\varepsilon_{n}^{i s}+k_{1} \Delta \varphi \varepsilon^{r} .
\end{aligned}
$$

Moreover, discretizing Eq. (9) and recasting Eq. (12) give:

$$
\left\{\begin{array}{l}
\varepsilon^{i \beta}=c_{\beta}^{-1}\left(\sigma+E_{\beta} b_{\beta} \varepsilon_{n}^{i \beta}\right) ; \quad \beta=r, g, h \\
\varepsilon^{i}=\varepsilon_{n}^{i}+b_{i} \sigma \\
\varepsilon^{i s}=k_{3}^{-1}\left(\varepsilon_{n}^{i s}+k_{4}\left(\left(b_{r}+1\right) \varepsilon^{i r}-b_{r} \varepsilon_{n}^{i r}\right)\right)
\end{array}\right.
$$

where:

$$
\begin{aligned}
& \left\{\begin{array}{l}
k_{3}=1-k_{2} \varphi^{-1} \Delta \varphi \\
k_{4}=k_{1} \Delta \varphi
\end{array}\right. \\
& b_{\beta}=\frac{\eta_{\beta}}{\Delta t E_{\beta}^{\text {neq }}}, \quad b_{i}=\frac{\Delta t}{\eta_{i}}, \quad c_{\beta}=E_{\beta} b_{\beta}+E_{\beta}^{\mathrm{eq}}, \\
& E_{\beta}=E_{\beta}^{\mathrm{eq}}+E_{\beta}^{\mathrm{neq}}, \quad \beta=r, g, h .
\end{aligned}
$$

Eqs. (12) to (14) show how internal variables are updated in $\Delta t$. Furthermore, after some mathematical manipulations, the axial stress is updated by Eq. (15) as shown in Box I. Eq. (15) can be recast as:

$$
\sigma=\chi_{1} \varepsilon^{m}+\chi_{2}
$$

in which parameters $\chi_{1}$ and $\chi_{2}$ are defined by Eq. (17) as shown in Box II. Eq. (16) in terms of the transverse deflection yields:

$$
\begin{aligned}
\sigma & =\chi_{1} \varepsilon^{m}+\chi_{2}=\chi_{1}\left(-y w^{\prime \prime}\right)+\chi_{2 y} y \\
& =y\left(-\chi_{1} w^{\prime \prime}+\chi_{2 y}\right),
\end{aligned}
$$

where prime stands for differentiation with respect to $x$. As observed in Eq. (18), the stress varies linearly within the beam height. Computing the moment about the $z$-axis, we may write:

$$
\begin{aligned}
M & =-\int_{A} \sigma y d A=-\int_{A}\left(-\chi_{1} w^{\prime \prime}+\chi_{2 y}\right) y^{2} d A \\
& =\left(\chi_{1} w^{\prime \prime}-\chi_{2 y}\right) I,
\end{aligned}
$$

in which $I$ and $M$ denote the moment of inertia and the moment along $z$-axis, respectively. $A$ also stands for cross sectional area of the beam. We now recast Eq. (19) as:

$$
M=\psi_{1} w^{\prime \prime}+\psi_{2}
$$

where:

$$
\psi_{1}=\chi_{1} I, \quad \psi_{2}=-\chi_{2 y} I
$$

$$
\sigma=\frac{\left[\begin{array}{l}
\left(\left[\varepsilon_{n}^{i s}+k_{3}\left(b_{h} \varepsilon_{n}^{i h}-b_{g} \varepsilon_{n}^{i g} \varphi\right)+b_{r} \varepsilon_{n}^{i r}\left(k_{3}(\varphi-1)-k_{4}\right)\right] \phi_{p}+\left(\varepsilon_{n}^{i}-b_{h} \varepsilon_{n}^{i h}-\varepsilon^{m}\right) k_{3}\right) c_{r} c_{g} c_{h} \\
+\left(c_{h}\left(b_{g}+1\right) b_{g} \varepsilon_{n}^{i g} E_{g} \phi_{p} \varphi+\left(1-\phi_{p}\right)\left(b_{h}+1\right) b_{h} \varepsilon_{n}^{i h} E_{h} c_{g}\right) c_{r} k_{3} \\
+\left(k_{3}+k_{4}-k_{3} \varphi\right)\left(b_{r}+1\right) b_{r} \phi_{p} \varepsilon_{n}^{i r} E_{r} c_{g} c_{h}
\end{array}\right]}{\left(\left(\left(1+b_{g}\right) \phi_{p} \varphi+a_{4} c_{g}\right) c_{h}+\left(b_{h}+1\right)\left(1-\phi_{p}\right) c_{g}\right) k_{3} c_{r}+\left(k_{3}(1-\varphi)+k_{4}\right)\left(b_{r}+1\right) \phi_{p} c_{g} c_{h}}
$$

where $\varepsilon^{m}$ denotes the mechanical part of the total strain $\left(\varepsilon^{m}=\varepsilon-\varepsilon^{T}\right)$. 


$$
\left\{\begin{array}{l}
\chi_{1}=\frac{c_{r} c_{g} c_{h} k_{3}}{\chi_{3}} \\
\chi_{2}=\frac{-1}{\chi_{3}}\left(\begin{array}{c}
\left(\begin{array}{c}
\varepsilon_{n}^{i s}+k_{3}\left(b_{h} \varepsilon_{n}^{i h}-b_{g} \varepsilon_{n}^{i g} \varphi\right) \\
+b_{r} \varepsilon_{n}^{i r}\left(k_{3}(\varphi-1)-k_{4}\right) \\
+\left(c_{h}\left(b_{g}+1\right) b_{g} \varepsilon_{n}^{i g} E_{g} \phi_{p} \varphi+\left(1-\phi_{p}\right)\left(b_{h}+1\right) b_{h} \varepsilon_{n}^{i h} E_{h} c_{g}\right) c_{r} k_{3} \\
+\left(k_{3}+k_{4}-k_{3} \varphi\right)\left(b_{r}+1\right) b_{r} \phi_{p} \varepsilon_{n}^{i r} E_{r} c_{g} c_{h}
\end{array}\right)=\chi_{2 y} y \\
\chi_{3}=\left(\left(\left(1+b_{g}\right) \phi_{p} \varphi+a_{4} c_{g}\right) c_{h}+\left(b_{h}+1\right)\left(1-\phi_{p}\right) c_{g}\right) k_{3} c_{r}+\left(k_{3}(1-\varphi)+k_{4}\right)\left(b_{r}+1\right) \phi_{p} c_{g} c_{h} c_{g} c_{h}
\end{array}\right.
\end{array}\right.
$$

Box II

The weak form is the result of the principle of virtual displacements. It states that if a body is in equilibrium, the total virtual work done by actual internal and external forces in moving through the virtual displacements is zero. This principle over a finite element $x \in\left[0, l^{e}\right]$, is:

$$
\delta W^{e}=\delta W^{E}+\delta W^{I}=0
$$

where $\delta W^{I}$ is the internal virtual work, and $\delta W^{E}$ is the external work done by the externally applied loads through the virtual displacements. The schematics of the beam element with the defined relevant degrees of freedom are shown in Figure 1.

The virtual work principle for the Euler-Bernoulli beam theory is reduced to:

$$
\delta W^{e}=\int_{A} \int_{0}^{l^{e}} \sigma \delta \varepsilon d x d A-\int_{0}^{l^{e}} q(x) \delta w d x-\sum_{i=1}^{4} Q_{i} \delta \Omega_{i},
$$

where $l^{e}$ is length of the element, $q(x)$ is the distributed transverse loads measured per unit length, $Q_{i}$ 's are the generalized nodal forces, and $\delta \Omega_{i}$ are the virtual generalized nodal displacements of the element. Thus, in light of definition (11), the virtual work statement in Eq. (21) becomes:

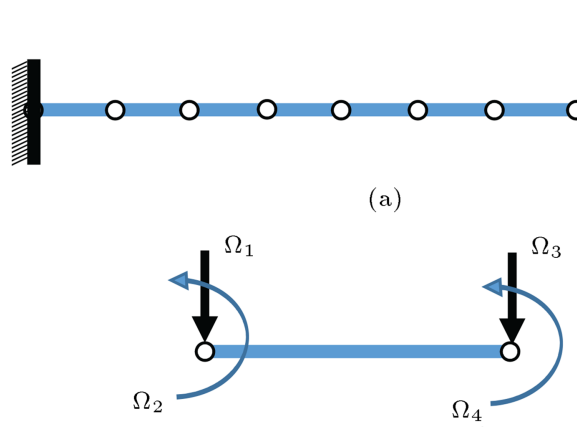

(b)

Figure 1. The schematics of the beam element together with the defined degrees of freedom.

$$
\begin{aligned}
& \delta \varepsilon=-y \frac{d^{2}(\delta w)}{d x^{2}} \\
& \Rightarrow \int_{A} \int_{0}^{l^{e}} \sigma \delta \varepsilon d x d A=-\int_{0}^{l^{e}} \frac{d^{2}(\delta w)}{d x^{2}} \overbrace{\int_{A} \sigma y d A}^{-M(x)} d x .
\end{aligned}
$$

Substituting Eq. (23) into Eq. (22) yields:

$$
\begin{aligned}
\delta W^{e}= & \int_{0}^{l^{e}} \frac{d^{2}(\delta w)}{d x^{2}} M(x) d x-\int_{0}^{l^{e}} q(x) \delta w d x \\
& -\sum_{i=1}^{4} Q_{i} \delta \Omega_{i}=0 .
\end{aligned}
$$

In light of Eqs. (20) and (24), we arrive at:

$$
\begin{aligned}
& \int_{0}^{l^{e}} \frac{d^{2}(\delta w)}{d x^{2}}\left[\psi_{1} \frac{d^{2} w}{d x^{2}}+\psi_{2}\right] d x-\int_{0}^{l^{e}} q(x) \delta w d x \\
& \quad-\sum_{i=1}^{4} Q_{i} \delta \Omega_{i}=0 .
\end{aligned}
$$

Employing 1D Hermite cubic interpolation function $N_{i}$, the transverse beam deflection is approximated in terms of four degrees of freedom, i.e. $\Omega_{i}$, as:

$$
w=\sum_{i=1}^{4} N_{i} \Omega_{i}, \quad \delta w=\sum_{i=1}^{4} N_{i} \delta \Omega_{i}
$$

where these degrees of freedom are:

$$
\begin{array}{ll}
\Omega_{1}=w(0), & \Omega_{2}=-d w /\left.d x\right|_{x=0}, \\
\Omega_{3}=w\left(l^{e}\right), & \Omega_{4}=-d w /\left.d x\right|_{x=l^{e}} .
\end{array}
$$

Substituting Eq. (26) into Eq. (25), after some mathematical manipulations, gives: 


$$
\begin{aligned}
\sum_{i=1}^{4} & \left(\int_{0}^{l^{e}} \frac{d^{2} N_{i}}{d x^{2}}\left[\psi_{1} \frac{d^{2} w}{d x^{2}}+\psi_{2}\right] d x\right. \\
& \left.-\int_{0}^{l^{e}} N_{i} q(x) d x-Q_{i}\right) \delta \Omega_{i}=0 .
\end{aligned}
$$

Assuming $w$ in the form of Eq. (26), Eq. (28) is rewritten as follows:

$$
\begin{aligned}
\int_{0}^{l^{e}} \frac{d^{2} N_{i}}{d x^{2}} & {\left[\psi_{1} \sum_{j=1}^{4} \frac{d^{2} N_{j}}{d x^{2}} \Omega_{j}+\psi_{2}\right] d x-\int_{0}^{l^{e}} q(x) N_{i} d x } \\
& -Q_{i}=0
\end{aligned}
$$

In order to present the matrix format for the element stiffness and load vectors, Eq. (29) is finally recast as:

$$
\left[K_{i j}\right]\left\{\Omega_{j}\right\}=\left\{F_{i}\right\}
$$

in which:

$$
\left\{\begin{array}{l}
K_{i j}=\psi_{1} \int_{0}^{l^{e}} \frac{d^{2} N_{i}}{d x^{2}} \frac{d^{2} N_{j}}{d x^{2}} d x \\
F_{i}=-\psi_{2} \int_{0}^{l^{e}} \frac{d^{2} N_{i}}{d x^{2}} d x+\int_{0}^{l^{e}} q(x) N_{i} d x+Q_{i}
\end{array}\right.
$$

For the sake of completeness, the Hermite shape functions are reported as follows:

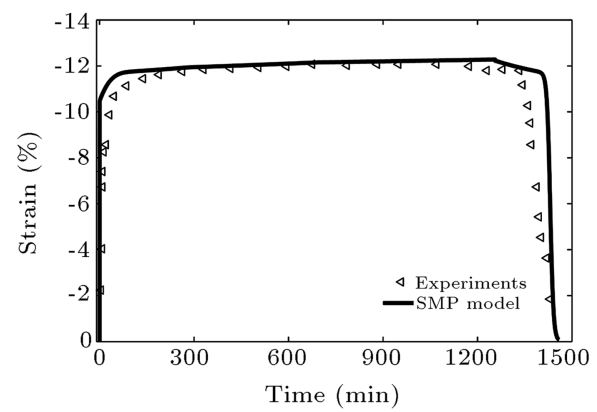

(a)

$$
\begin{array}{ll}
N_{1}=\frac{2 x^{3}-3 x^{2} l^{e}+l^{e^{3}}}{l^{e^{3}}}, & N_{2}=\frac{x^{3} l^{e}-2 x^{2} l^{e^{2}}+x l^{e^{3}}}{l^{e^{3}}}, \\
N_{3}=\frac{-2 x^{3}+3 x^{2} l^{e}}{l^{e^{3}}}, & N_{4}=\frac{x^{3} l^{e}-x^{2} l^{e^{2}}}{l^{e^{3}}} .
\end{array}
$$

It is finally reminded that $\psi_{1}$ and $\psi_{2}$ are memory parameters. These parameters are updated in each increment and are responsible for the history dependency of the SMP element response, as described in Eqs. (17) and (20).

\section{Numerical results}

To show validity of the model in time-dependent regimes of thermomechanical loadings, we first report the simulation results according to experiments performed in [43] already published in [38]. At the start of the experiment, an SMP-based syntactic foam sample is compressed under a constant stress $\sigma_{0}=$ $-263 \mathrm{kPa}$ at $T_{h}$ for 30 minutes. Keeping the stress fixed, the temperature is lowered to $T_{l}$. The cooling rate is found through the Newton's law of cooling: $\frac{d T}{d t}=4.6 \times 10^{-5}(T-293)[38]$. Once the temperature reaches $T_{l}$ (at $t=1256 \mathrm{~min}$ ), the system is unloaded and, then, it is heated up to $T_{h}$ with heating rate $=0.3 \frac{\mathrm{K}}{\min }$. Therequired material parameters are tabulated in Table 1. The strain-time behavior is shown in Figure 2(a). A good correlation is observed between the experiments and the numerical results. If the recovery occurs in a fixed-strain stress recovery, the stress-temperature behavior is found as shown in

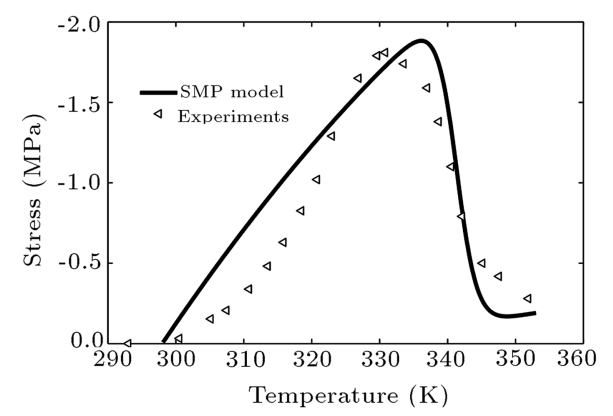

(b)

Figure 2. Reproduction of the shape-memory effect [38]: (a) Stress-free strain recovery and (b) fixed-strain stress recovery. Experiments are reported in [43].

Table 1. Material parameters reported by Baghani et al. [38] adopted from experiments conducted in [43].

\begin{tabular}{ccc}
\hline Material parameters & Values & Units \\
\hline$E_{r}^{\mathrm{eq}}, E_{g}^{\mathrm{eq}}, E_{h}^{\mathrm{eq}}, E_{r}^{\text {neq }}, E_{g}^{\text {neq }}, E_{h}^{\text {neq }}$ & $1.3,15,70000,0.2,247,1000$ & $(\mathrm{MPa})$ \\
$\eta_{r}, \eta_{g}, \eta_{h}$ & $5,30 \times 10^{6}, 30 \times 10^{9}$ & $($ MPa.min $)$ \\
$\nu_{r}, \nu_{g}, \nu_{h}$ & $0.49,0.3,0.3$ & $(-)$ \\
$T_{l}, T_{g}, T_{h}$ & $296,344,353$ & $(\mathrm{~K})$ \\
$\varepsilon^{T}$ & $\left(0.5542 \times 10^{-3} T-0.01083-7 \times 10^{-7} T^{2}\right)$ & $(-)$ \\
$\varphi_{g}$ & $1-\frac{1}{1+\exp \left(-0.66\left(T-T_{g}\right)\right)}$ & $(-)$ \\
\hline
\end{tabular}


Figure 2(b). These results show that the SMP model is capable of predicting the main governing phenomena in different SME cycles.

Now, we employ the presented SMP beam element to investigate the bending of a cantilever SMP beam, experiencing a stress-free strain recovery cycle. An I-shaped SMP beam is selected for presenting the numerical results. Thickness of the beam cross section is $1 \mathrm{~mm}$ and its height and width are $10 \mathrm{~mm}$ and $12 \mathrm{~mm}$, respectively. The history of the applied force on the free tip of the beam, as well as the temperature history, is shown in Figure 3. Making

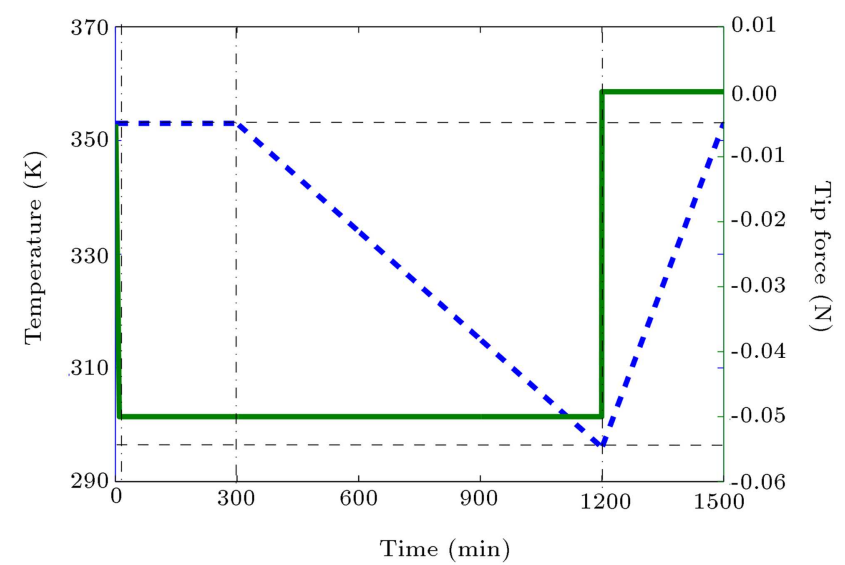

Figure 3. History of the force and temperature applied to the beam. use of the material parameters reported in Table 1, the corresponding results are illustrated in Figure 4. In order to validate results of the present work, the 3D SMP constitutive model is implemented in a finite element framework. For 3D simulations, we use the nonlinear finite element software ABAQUS/Standard, implementing the described algorithm in [38] within a user-defined subroutine UMAT. Employing this 3D finite element tool, the whole SME cycle is simulated. In Figure 4(a), the free tip displacement results of the SMP beam model (present work) together with those of the 3D modeling are depicted. As one may observe, at different values of $\phi_{h}$, an acceptable correspondence is seen between the results of these two methods. Moreover, at larger values of $\phi_{h}$, as expected, the structure becomes stiffer. Hence, smaller beam tip deflections are predicted. Besides, as observed in Figure 4(a), the beam is capable of complete recovery of the shape. It can be justified considering that in this example, $\eta_{i}$ has a very large value. As a result, no residual irreversible strain remains in the structure. The time-dependent viscoelatic behavior of the structure at temperatures higher than $T_{h}$ is clearly seen in Figure 4(a).

In Figure 4(b), the variations of axial strain at upper surface of the beam root (the most critical point of the structure) are plotted. This figure shows that the maximum strain is about $3.3 \%$. Thus, the small

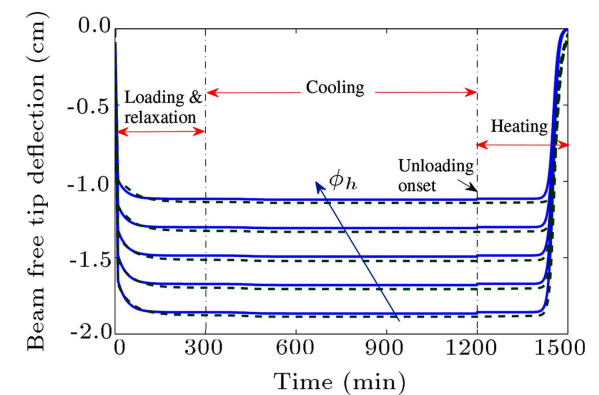

(a)

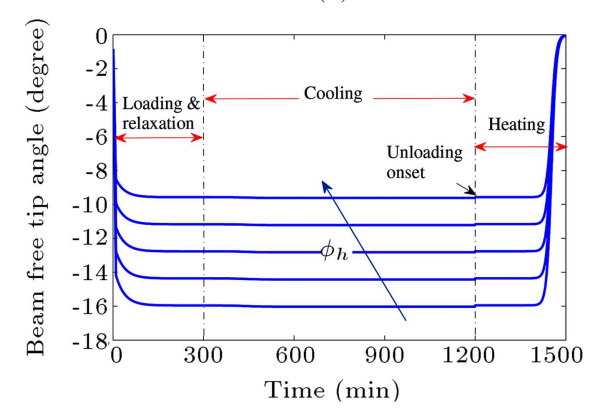

(c)

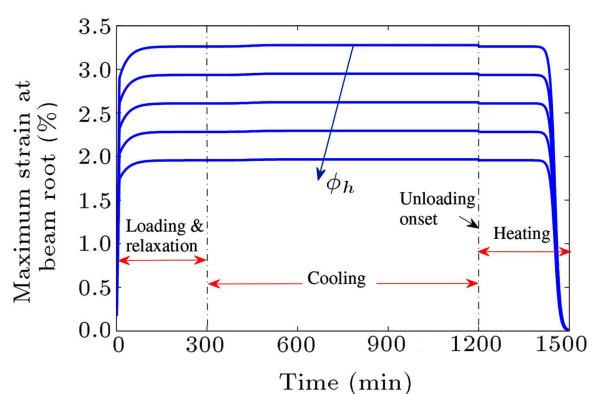

(b)

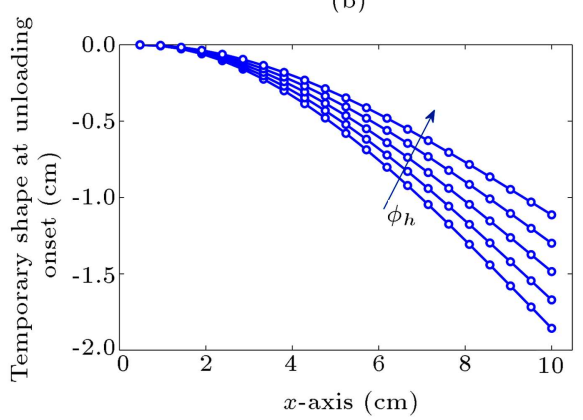

(d)

Figure 4. Effect of $\phi_{h}$ on (a) the free tip displacement results of the SMP beam model (present work) together with those of the 3D modeling (solid lines show the beam element results, while dashed-lines represent the 3D finite element predictions), (b) variation of axial strain at upper surface of the beam root (the most critical point of the structure), (c) the free tip angle of the beam, and $(\mathrm{d})$ the temporarily memorized shape of the beam (after unloading). $F_{\max }=-0.05 \mathrm{~N}$, $q=0(\mathrm{~N} / \mathrm{m})$, and $\phi_{h}=0,0.1,0.2,0.3,0.4$. 
strain assumption used in developing the element is not violated. Moreover, in Figure 4(c), the free tip angle of the beam is shown. This figure also shows the maximum angle smaller than 16 degrees. Thus, the assumption of small rotations also stands. In Figure $4(d)$, the temporary shape of the beam (after unloading) is depicted at different $\phi_{h}$ 's.

In order to investigate the effects of different values of viscosity parameters, in Figure 5, the free tip displacement results of the SMP beam model are depicted. It is shown that increasing $\eta_{r}$ makes the structure need more time to react against the applied stress. To investigate the effect of applying an external force during the heating process on the shape recovery, free tip of the beam is plotted in Figure 6. In this figure, $F_{\max }$ is the maximum force applied to free tip of the beam in the loading path and $F_{h}$ is the force applied to the beam within the heating process. As observed in Figure 6, without any external force $\left(F_{h}=0\right)$, the SMP beam fully recovers its original shape, while increasing the external force (during the heating) results in smaller values of recovery ratio as illustrated in Figure 6 . In the special case where $F_{h}=$ $F_{\max }$, we almost have no recovery. It shows that after heating recovery, stored strains completely vanish and responses of the structure to the mechanical loadings are similar to the first responses, i.e. those at $T_{h}$.

The developed formulation can be used in any

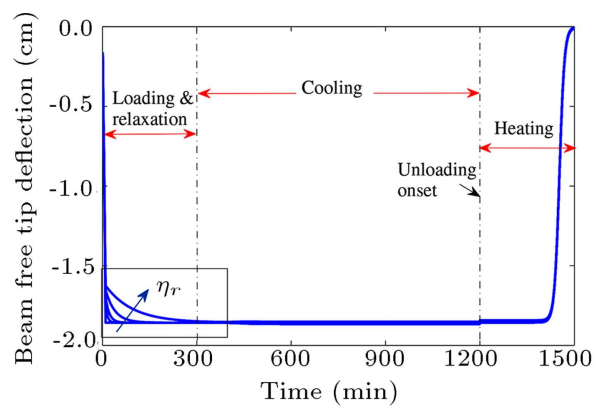

(a)
Table 2. SMP behavior of theophylline-loaded PCL/TspPOSS (90/10) nanocomposites [25].

\begin{tabular}{ccc}
\hline $\begin{array}{c}\text { Theophylline } \\
\text { weight (\%) }\end{array}$ & $\begin{array}{c}\text { Shape } \\
\text { fixity (\%) }\end{array}$ & $\begin{array}{c}\text { Shape } \\
\text { recovery (\%) }\end{array}$ \\
\hline 0 & 85 & 94 \\
5 & 82 & 87 \\
10 & 81 & 74 \\
\hline
\end{tabular}

structure that undergoes bending. If we want to properly model a drug in which an SMP-nanocomposite is coated on the drug, e.g. theophylline, we should just use the SMP beam element developed here, accompanied by elastic/hyperelastic/viscoelastic elements (these elements stand for the drug, e.g. theophylline parts). In Table 2, the recovery and shape fixity ratios of the SMP-nanocomposite drug are tabulated. These data are borrowed from [25]. As one may observe in this table, increasing the weight fraction of theophylline leads to a decrease in the shape recovery as well as the shape fixity of the drug. This trend is qualitatively in agreement with those found in this paper.

\section{Summary and conclusions}

In this work, by employing a new constitutive model recently proposed for SMPs, a new SMP beam element was developed in a finite element framework to predict the response of reinforced SMP beams under

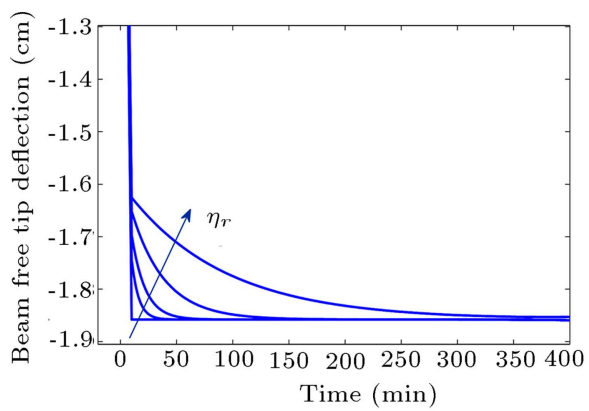

(b)

Figure 5. (a) Effect of $\eta_{r}$ on the free tip displacement results of the SMP beam model $\left(\eta_{r}=0,1,2,5,10 \mathrm{MPa}\right.$.min and $\phi_{h}=0$ ). (b) The rectangle in part (a) is zoomed in.

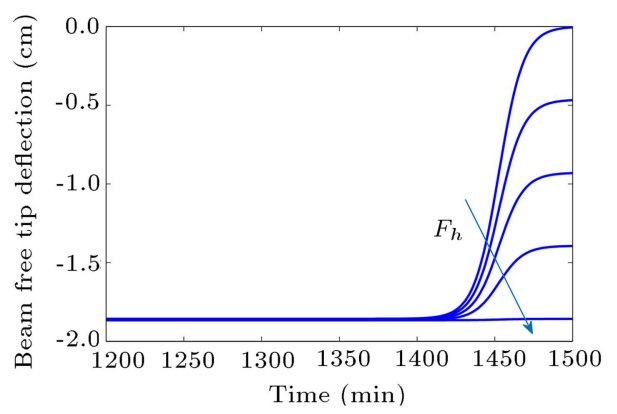

(a)

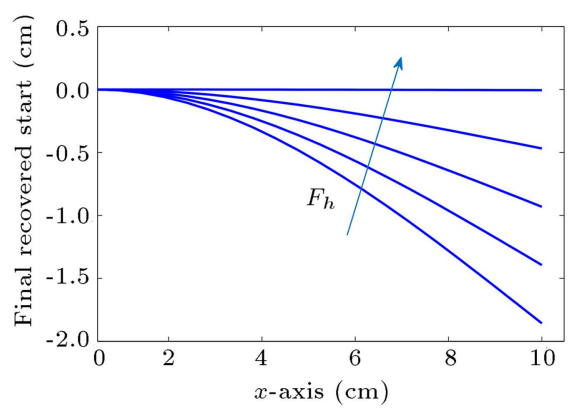

(b)

Figure 6. Effect of applying an external force during the heating process to the shape recovery on (a) the free tip displacement and (b) the recovered shape $\left(F_{h} / F_{\max }=0.0,0.1,0.2,0.5,1.0\right.$ and $\left.\phi_{h}=0\right)$. 
bending in thermo-mechanical cycles. To develop the formulations, the assumptions of Euler-Bernoulli beam theory were employed. It was observed that within the cooling (shape fixation) process, the stored strains evolved to fix the temporary shape; then, during the heating process, these strains started to relax, in order to recover the original shape. Effects of different values of hard segment volume fraction on the SMP beam response for a cantilever reinforced SMP beam were also investigated. It was observed that increasing volume fraction of hard segment acted as reinforcement and made the structure stiffer. Thus, as a conclusion, larger values of external loads could be handled. Moreover, the time-dependent effects were investigated through a parametric study on the viscosity coefficient in the rubbery phase. It was shown that in larger values of $\eta_{r}$, the structure needed more time to arrive at a steady state. With no external forces within the heating step, the SMP beam almost fully recovered its original shape, while increasing the external force (during heating) resulted in smaller values of recovery ratio. In the special case that the external loadings were same as the loadings in the loading step, the structure experienced almost no shape recovery. Comparing the numerical results with those of $3 \mathrm{D}$ finite element modeling ensures the validity of the beam element developments and the assumptions made therein. The proposed finite element beam formulation can be used as an efficient tool for investigating the effects of changing any material or geometrical parameter on reinforced SMP beams, where we need a large number of simulations and 3D modeling is computationally too expensive. As an application, it was shown that the developed nanocomposite SMP beam element could be used for numerical modeling of thermomechanical response of the drugs (e.g., theophylline) coated by films of SMP nanocomposites. It was observed that the numerical results were qualitatively in correspondence with those of experiments reported for sustained release of SMPnanocomposite based drugs already available in the literature.

\section{References}

1. Schmidt, C., Chowdhury, A.M.S., Neuking, K. and Eggeler, G. "Mechanical behavior of shape memory polymers by $1 \mathrm{WE}$ method: Application to tecoflex", Journal of Thermoplastic Composite Materials, 24, pp. 853-860 (2011).

2. Hashemi, S.M.T. and Khadem, S.E. "Modeling and analysis of the vibration behavior of a shape memory alloy beam", International Journal of Mechanical Sciences, 48, pp. 44-52 (2006).

3. Zbiciak, A. "Dynamic analysis of pseudoelastic sma beam", International Journal of Mechanical Sciences, 52, pp. 56-64 (2010).
4. Lendlein, A. and Behl, M. "Shape-memory polymers for biomedical applications", Advances in Science and Technology, 54, pp. 96-102 (2009).

5. Ghosh, P., Reddy, J.N. and Srinivasa, A.R. "Development and implementation of a beam theory model for shape memory polymers", International Journal of Solids and Structures, 50, pp. 595-608 (2012).

6. Lendlein, A. and Langer, R. "Biodegradable, elastic shape-memory polymers for potential biomedical applications", Science, 296, pp. 1673-1676 (2002).

7. Gall, K., Dunn, M., Liu, Y., Stefanic, G. and Balzar, D. "Internal stress storage in shape memory polymer nanocomposites", Applied Physics Letters, 85, pp. 290292 (2004).

8. Diani, J., Liu, Y. and Gall, K. "Finite strain 3D thermoviscoelastic constitutive model for shape memory polymers", Polymer Engineering and Science, 46, pp. 486-492 (2006).

9. Leng, J., Lan, X., Liu, Y. and Du, S. "Shape-memory polymers and their composites: Stimulus methods and applications", Progress in Materials Science, 56, pp. 1077-1135 (2011).

10. Leng, J., Lv, H., Liu, Y. and Du, S. "Electroactivate shape-memory polymer filled with nanocarbon particles and short carbon fibers", Applied Physics Letters, 91, p. 144105 (2007).

11. Xu, W. and Li, G. "Constitutive modeling of shape memory polymer based self-healing syntactic foam", International Journal of Solids and Structures, 47, pp. 1306-1316 (2010).

12. Lan, X., Liu, L., Liu, Y., Leng, J. and Du, S. "Post microbuckling mechanics of fibre-reinforced shapememory polymers undergoing flexure deformation", Mechanics of Materials, 72, pp. 46-60 (2014).

13. Qiao, T., Liu, L., Liu, Y. and Leng, J. "Post buckling analysis of the shape memory polymer composite laminate bonded with alloy film", Composites Part B: Engineering, 53, pp. 218-225 (2013).

14. Lan, X., Liu, Y., Lv, H., Wang, X., Leng, J. and Du, S. "Fiber reinforced shape-memory polymer composite and its application in a deployable hinge", Smart Materials and Structures, 18, p. 024002 (2009).

15. Lu, H., Yu, K., Liu, Y. and Leng, J. "Sensing and actuating capabilities of a shape memory polymer composite integrated with hybrid filler", Smart Materials and Structures, 19, p. 065014 (2010).

16. Mohamadpour, S., Pourabbas, B. and Fabbri, P. "Anti-scratch and adhesion properties of photocurable polymer/clay nanocomposite coatings based on methacrylate monomers", Scientia Iranica, 18, pp. 765-771 (2011).

17. Hajiali, H., Karbasi, S., Hosseinalipour, M. and Rezaie, H. "Effects of bioglass nanoparticles on bioactivity and mechanical property of poly (3-hydroxybutirate) scaffolds", Scientia Iranica, Transactions F, Nanotechnology, 20, p. 2306 (2013). 
18. Yanju, L., Haiyang, D., Liwu, L. and Jinsong, L. "Shape memory polymers and their composites in aerospace applications: a review", Smart Materials and Structures, 23, p. 023001 (2014).

19. Liu, Y., Lv, H., Lan, X., Leng, J. and Du, S. "Review of electro-active shape-memory polymer composite", Composites Science and Technology, 69, pp. 2064-2068 (2009).

20. Zhou, B., Liu, Y.J., Lan, X., Leng, J.S. and Yoon, S.H. "A glass transition model for shape memory polymer and its composite", International Journal of Modern Physics B, 23, pp. 1248-1253 (2009).

21. Lendlein, A. and Langer, R. Biodegradable "Elastic shape-memory polymers for potential biomedicala pplications", Science, 296, pp. 1673-1676 (2002).

22. Mohamed, F. and Van Der Walle, C.F. "Engineering biodegradable polyester particles with specific drug targeting and drug release properties", Journal of Pharmaceutical Sciences, 97, pp. 71-87 (2008).

23. Neffe, A.T., Hanh, B.D., Steuer, S. and Lendlein, A. "Polymer networks combining controlled drug release, biodegradation, and shape memory capability", Advanced Materials, 21, pp. 3394-3398 (2009).

24. Hetrick, E.M. and Schoenfisch, M.H. "Reducing implant-related infections: Active release strategies", Chemical Society Reviews, 35, pp. 780-7891,1 (2006).

25. Kashif, M., Yun, B.M., Lee, K.S. and Chang, Y.W. "Biodegradable shape-memory poly (caprolactone)/polyhedral oligomeric silsequioxane nanocomposites: Sustained drug release and hydrolytic degradation", Materials Letters, 166, pp. 125-128 (2016).

26. Lu, H., Liu, J., Zhu, S., Yang, Y. and Fu, Y.Q. "Enhanced electro-activated performance of shape memory polymer nanocomposites with self-assembled carbon nanofibre template", Nanoscience and Nanotechnology Letters, 7, pp. 94-99 (2015).

27. Shen, H., Mark, A., Thompson, K., Xu, Y., Liang, F., Gou, J. and Mabbott, B. "Thermal modeling and coefficient identification of shape memory polymer nanocomposites structure", Applied Physics Letters, 106, p. 081907 (2015).

28. Zhang, C.S. and Ni, Q.Q. "Bending behavior of shape memory polymer based laminates", Composite Structures, 78, pp. 153-161 (2007).

29. Liu, Y., Gall, K., Dunn, M.L. and McCluskey, P. "Thermomechanical recovery couplings of shape memory polymers in flexure", Smart Materials and Structures, 12, pp. 947-954 (2003).

30. Tobushi, H., Hayashi, S., Hoshio, K. and Ejiri, Y. "Shape recovery and irrecoverable strain control in polyurethane shape-memory polymer", Science and Technology of Advanced Materials, 9, p. 015009 (2008).

31. Liu, Y., Gall, K., Dunn, M., Greenberg, A. and Diani, J. "Thermomechanics of shape memory polymers: Uniaxial experiments and constitutive modeling", International Journal of Plasticity, 22, pp. 279313 (2006).
32. Atli, B., Gandhi, F. and Karst, G. "Thermomechanical characterization of shape memory polymers", Journal of Intelligent Material Systems and Structures, 20, pp. 87-95 (2009).

33. Kim, J., Kang, T. and Yu, W. "Thermo-mechanical constitutive modeling of shape memory polyurethanes using a phenomenological approach", International Journal of Plasticity, 26, pp. 204-218 (2010).

34. Tobushi, H., Okumura, K., Hayashi, S. and Ito, N. "Thermomechanical constitutive model of shape memory polymer", Mechanics of Materials, 33, pp. $545-554$ (2001).

35. Baghani, M., Mohammadi, H. and Naghdabadi, R. "An analytical solution for shape-memory-polymer euler-bernoulli beams under bending", International Journal of Mechanical Sciences, 84, p. 8490 (2014).

36. Baghani, M., Naghdabadi, R., Arghavani, J. and Sohrabpour, S. "A constitutive model for shape memory polymers with application to torsion of prismatic bars", Journal of Intelligent Material Systems and Structures, 23, pp. 107-116 (2012).

37. Baghani, M. and Taheri, A. "An analytic investigation on behavior of smart devices consisting of reinforced shape memory polymer beams", Journal of Intelligent Material Systems and Structures, p. 1045389X14541503 (2014).

38. Baghani, M., Naghdabadi, R., Arghavani, J. and Sohrabpour, S. "A thermodynamically-consistent 3D constitutive model for shape memory polymers", International Journal of Plasticity, 35, pp. 13-30 (2012).

39. Baghani, M., Naghdabadi, R. and Arghavani, J. "A semi-analytical study on helical springs made of shape memory polymer", Smart Materials and Structures, 21, p. 045014 (2012).

40. Baghani, M., Naghdabadi, R. and Arghavani, J. "A large deformation framework for shape memory polymers: Constitutive modeling and finite element implementation", Journal of Intelligent Material Systems and Structures, 24, pp. 21-32 (2013).

41. Baghani, M., Arghavani, J. and Naghdabadi, R. "A finite deformation constitutive model for shape memory polymers based on Hencky strain", Mechanics of Materials, 73, pp. 1-10 (2014).

42. Reddy, J.N., An Introduction to Nonlinear Finite Element Analysis: With Applications to Heat Transfer, Fluid Mechanics, and Solid Mechanics, OUP Oxford (2014).

43. Li, G. and Nettles, D. "Thermomechanical characterization of a shape memory polymer based selfrepairing syntactic foam", Polymer, 51, pp. 755-762 (2014).

\section{Biographies}

Mostafa Baghani received his BS degree in Mechanical Engineering from University of Tehran, Iran, 
in 2006 and his MS and $\mathrm{PhD}$ degrees in Mechanical Engineering from the Department of Mechanical Engineering at Sharif University of Technology, Tehran, Iran, in 2008 and 2012, respectively. He is now an Assistant Professor in the School of Mechanical Engineering, University of Tehran. His research interests include solid mechanics, nonlinear finite element method, and shape-memory materials constitutive modeling.

Roshanak Dolatabadi received her PharmD degree from Mashhad University of Medical Sciences, Iran, in 2014. She is now a PhD student in Department of Drug and Food Control, Faculty of Pharmacy, Tehran
University of Medical Sciences, Tehran, Iran. Her research interests are pharmaceutics and designing new techniques for release profile controllability of drugs through methods such as extrusion-spheronization, pelletization, coating, etc.

Majid Baniassadi is working as Assistant Professor in the Faculty of Mechanical Engineering at University of Tehran. He received his BS degree from Isfahan University of Technology, in 2004, MSc degree from University of Tehran in 2006, and PhD from University of Strasbourg in 2011. His research interests are mainly in the area of micromechanics, nano-mechanics, and materials design. 